\title{
Finding large matchings in 1-planar graphs of minimum degree 3
}

\author{
Therese Biedl * $\quad$ Fabian Klute $^{\dagger}$
}

\begin{abstract}
A matching is a set of edges without common endpoint. It was recently shown that every 1-planar graph (i.e., a graph that can be drawn in the plane with at most one crossing per edge) that has minimum degree 3 has a matching of size at least $\frac{n+12}{7}$, and this is tight for some graphs. The proof did not come with an algorithm to find the matching more efficiently than a general-purpose maximum-matching algorithm. In this paper, we give such an algorithm. More generally, we show that any matching that has no augmenting paths of length 9 or less has size at least $\frac{n+12}{7}$ in a 1 -planar graph with minimum degree 3 .
\end{abstract}

\section{Introduction}

The matching problem (i.e., finding a large set of edges in a graph such that no two chosen edges have a common endpoint) is one of the oldest problem in graph theory and graph algorithms, see for example [3, 18] for overviews.

To find the maximum matching in a graph $G=(V, E)$, the fastest algorithm is the one by Hopcroft and Karp if $G$ is bipartite [15], and the one by Micali and Vazirani otherwise (19], see also [24] for further clarifications). As pointed out in [24], for a graph with $n$ vertices and $m$ edges the run-time of the algorithm by Micali and Vazirani is $O(m \sqrt{n})$ in the RAM model and $O(m \sqrt{n} \alpha(m, n))$ in the pointer model, where $\alpha(\cdot)$ is the inverse Ackerman function. For planar graphs (graphs that can be drawn without crossing in the plane) there exists a linear-time approximation scheme for maximum matching [1, and it can easily be generalized to so-called $H$-minor-free graphs [9] and $k$-planar graphs [13].

For many graph classes, specialized results concerning matchings and matching algorithms have been found. To name just a few, every bipartite $d$-regular graph has a perfect matching (a matching of size $n / 2$ ) [14] and it can be found in $O(m \log d)$ time [8]. Every 3-regular biconnected graph has a perfect matching [21 and it can be found in linear time

${ }^{*}$ David R. Cheriton School of Computer Science, University of Waterloo, Waterloo, Ontario N2L 1A2, Canada. Supported by NSERC. biedl@uwaterloo.ca

$\dagger^{\dagger}$ Algorithms and Complexity Group, TU Wien, Austria, fklute@ac.tuwien.ac.at. Research initiated while the author was visiting the University of Waterloo. 
for planar graphs and in near-linear time for arbitrary graphs [4]. Every graph with a Hamiltonian path has a near-perfect matching (of size $\lceil(n-1) / 2\rceil$ ); this includes for example the 4-connected planar graphs [23] for which the Hamiltonian path (and with it the near-perfect matching) can be found in linear time [7].

For graphs that do not have perfect or near-perfect matchings, one possible avenue of exploration is to ask for guarantees on the size of matchings. One of the first results in this direction is due to Nishizeki and Baybars [20], who showed that every planar graph with minimum degree 3 has a matching of size at least $\frac{n+4}{3}$. (This bound is tight for some planar graphs with minimum degree 3.) The proof relies on the Tutte-Berge theorem and does not give an algorithm to find such a matching (or at least, none faster than any maximummatching algorithm). Over 30 years later, a linear-time algorithm to find a matching of this size in planar graphs of minimum degree 3 was finally developed by Franke, Rutter and Wagner [12]. The latter paper was a major inspiration for our current work.

In recent years, there has been much interest in near-planar graphs, i.e., graphs that may be required to have crossings but that are "close" to planar graphs in some sense. We are interested here in 1-planar graphs, which are those that can be drawn with at most one crossing per edge. (Detailed definitions can be found in Section 2, See a recent annotated bibliography [17] for an overview of many results known for 1-planar graphs. The first author and Wittnebel [5] gave matching-bounds for 1-planar graphs of varying minimum degrees, and showed that any 1-planar graph with minimum degree 3 has a matching of size at least $\frac{n+12}{7}$. (Again, this bound is tight for some 1-planar graphs with minimum degree 3.)

The proof in [5] is again via the Tutte-Berge theorem and does not give rise to a fast algorithm to find a matching of this size. This is the topic of the current paper. We give an algorithm that finds, for any 1-planar graph with minimum degree 3, a matching of size at least $\frac{n+12}{7}$ in linear time in the RAM model and time $O(n \alpha(n))$ in the pointer-model. The algorithm consists simply of running the algorithm by Micali and Vazirani for a limited number of rounds (and in particular, does not require that a 1-planar drawing of the graph is given). The bulk of the work consists of the analysis, which states that if there are no augmenting paths of length 9 or less, then the matching has the desired size for graphs with minimum degree 3. Along the way, we also prove some bounds obtained for graphs with higher minimum degree, though these are not tight.

The paper is structured as follows. After reviewing some background in Section 2, we state the algorithm in Section 3. The analysis proceeds in multiple steps in Section 4 . We first delete short flowers from the graph (and account for free vertices in them directly). The remaining graph is basically bipartite, and we can use bounds known for independent sets in 1-planar graphs to obtain matching-bounds that are very close to the desired goal. Closing this gap requires a non-trivial modification of the graph and argument; this is deferred to Section 5 before we conclude in Section 6 . 


\section{Background}

We assume familiarity with graphs and graph algorithms, see for example [10] and [22]. Throughout the paper, $G$ is a simple graph with $n$ vertices and $m$ edges. A matching is a set $M$ of edges without common endpoints; we say that $e=(x, y) \in M$ is matched and $x$ and $y$ are matching-partners. $V(M)$ denotes the endpoints of edges in $M$; we call $v \in V(M)$ matched and all other vertices free. An alternating walk is a walk that alternates between unmatched and matched edges. An augmenting path is an alternating walk that repeats no vertices and begins and ends at a free vertex; we use $k$-augmenting path for an augmenting path with at most $k$ edges. Note that if $P$ is an augmenting path of $M$ (and viewed as an edge-set), then $(M \backslash P) \cup(P \backslash M)$ is also a matching and has one more edge.

A drawing $\Gamma$ of a graph consists of assigning points in $\mathbb{R}^{2}$ to vertices and simple curves to each edge such that curves of edges end at the points of its endpoints. We usually identify the graph-theoretic object (vertex, edge) with the geometric object (point, curve) that it has been assigned to. A drawing is called good (see [22] for details) if (1) no edge intersects a point of a non-incident vertex, (2) two edges intersect in at most We only consider good drawings (see 22] for details) that avoid degeneracies such an edge going through the point of a non-incident vertex or two edges with a common endpoint intersecting. The connected regions of $\mathbb{R}^{2} \backslash \Gamma$ are called the regions of the drawing.

A crossing $c$ of $\Gamma$ is a pair of two edges $(v, w)$ and $(x, y)$ that have a point in their interior in common. A crossed edge is one that has a crossing on it; otherwise it is called uncrossed. A drawing $\Gamma$ is called $k$-planar (or planar for $k=0$ ) if every edge has at most $k$ crossings. A graph is called $k$-planar if it has a $k$-planar drawing. While planarity can be tested in linear time [16, 6], testing 1-planarity is NP-hard [13.

Fix a 1-planar drawing $\Gamma$ and consider a crossing $c$ between edges $\left(v_{0}, v_{2}\right)$ and $\left(v_{1}, v_{3}\right)$. Then we could draw edge $\left(v_{i}, v_{i+1}\right)$ (for $i=0, \ldots, 3$ and addition modulo 4 ) without crossing by walking "very close" to crossing $c$. We call the pair $\left(v_{i}, v_{i+1}\right)$ a potential kite-edge and note that if we inserted $\left(v_{i}, v_{i+1}\right)$ in the aforementioned manner, then it would be consecutive with the crossing edges in the cyclic orders of edges around $v_{i}$ and $v_{i+1}$ in $\Gamma$.

\section{Finding the matching}

Our algorithm to find a large matching is a one-liner: repeatedly extend the matching via 9-augmenting paths (i.e., of length at most 9) until there are no more such paths.

Note that the algorithm does not depend on the knowledge that the graph is 1-planar and does not require having a 1-planar drawing at hand. It could be executed on any graph; our contribution is to show (in the next section) that if it is executed on a 1-planar graph $G$ with minimum degree 3 then the resulting matching has size at least $\frac{n+12}{7}$.

Running time Finding a matching $M$ in $G$ such that there is no $k$-augmenting path can be done in time $O(k|E|)$ using the algorithm by Micali and Vazirani [19]. (We state all run-time bounds here in the RAM model; for the pointer model add a factor of $\alpha(|E|,|V|)$.) 
This algorithm runs in phases, each of which has a running time of $O(|E|)$ and increases the length of the minimum-length augmenting path by at least two. See for example the paper by Bast et al. [2] for a more detailed explanation. Since for 1-planar graphs we have $|E| \in O(|V|)$ we get a linear time algorithm in the number of vertices of $G$ to find a matching without 9-augmenting paths.

\section{Analysis}

Assume that $M$ is a matching without augmenting paths of length at most 9 , and let $F$ be the free vertices; $|F|=n-2|M|$. To analyze the size of $M$, we proceed in three stages. First we remove some vertices and matching-edges that belong to short flowers (defined below); these are "easy" to account for. Next we split the remaining vertices by their distance (measured along alternating paths) to free vertices. Since short flowers have been removed, no edges can exist between vertices of even small distance; they hence form an independent set. Using a crucial lemma from [5] on the size of independent sets in 1-planar graphs, this shows that $|M| \geq \frac{7}{50}(n+12)$, which is very close to the desired bound of $\frac{n+12}{7}$. The last stage (which does the improvement from $\frac{7}{50}$ to $\frac{1}{7}$ ) will require non-trivial effort and is done mostly out of academic interest; this is deferred to Section 5 .

Flowers A flower ${ }^{1}$ is an alternating walk that begins and ends at the same free vertex; we write $k$-flower for a flower with at most $k$ edges. We only consider 7 -flowers; Figure 1 illustrates all possible such flowers. Note that such short flowers split into a path (called stem) and an odd cycle (the blossom); we call a flower a cycle-flower if the stem is empty.
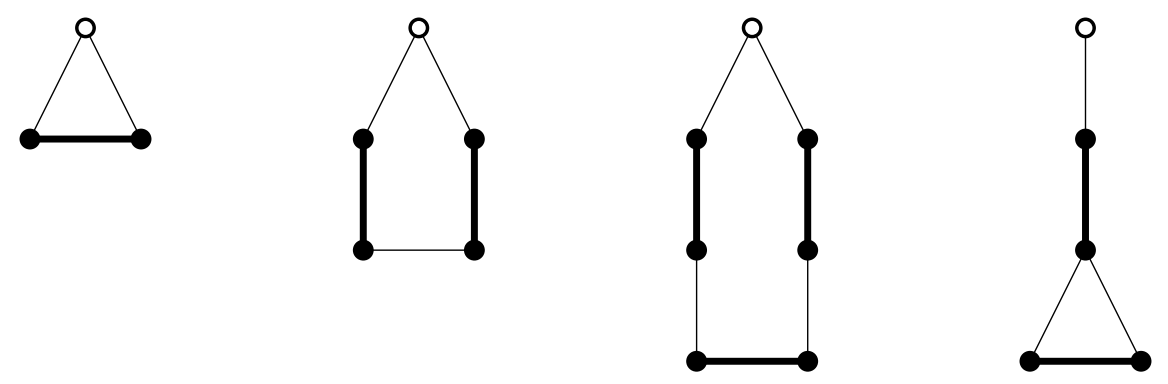

Figure 1: All possible flowers of length up to 7. Free vertices are white, matched edges are thick.

Let $V_{C}$ (the "C" reminds of "cycle") be all vertices that belong to a 7-cycle-flower and let $M_{C}$ and $F_{C}$ be all matching-edges and free vertices within $V_{C}$.

Claim 1. $\left|F_{C}\right| \leq\left|M_{C}\right|$.

Proof. For every $f \in F_{C}$ there exists some 7-cycle-flower $f-v_{1}-v_{2}-\ldots-v_{k^{-}} f$ with $k \in\{2,4,6\}$. Assign $f$ to matching-edge $\left(v_{1}, v_{2}\right) \in M_{C}$. Assume for contradiction that another vertex

\footnotetext{
${ }^{1}$ Our terminology follows the one in Edmonds' famous blossom-algorithm [11].
} 
$f^{\prime} \in F_{C}$ was also assigned to $\left(v_{1}, v_{2}\right)$. Then $f^{\prime}$ is adjacent to one of $v_{1}, v_{2}$. If it is $v_{2}$, then $f^{\prime}-v_{2}-v_{1}-f$ is a 3 -augmenting path. If it is $v_{1}$, then $f^{\prime}-v_{1}-\ldots-v_{k^{-}} f$ is a 7 -augmenting path, see Figure 2(a).

From now on we will only study the graph $G \backslash V_{C}$. Let $F_{B}$ (the " $B$ " reminds of "blossom") be all those free vertices $f$ that are not in $F_{C}$ and that belong to a 7-flower. By $f \notin F_{C}$ this flower has a non-empty stem, which is possible only if its length is exactly 7 and the stem has two edges $f$-s- $t$ while the blossom is a 3 -cycle $t$ - $x_{0}-x_{1}-t$. Furthermore $(s, t)$ and $\left(x_{0}, x_{1}\right)$ are matching-edges. Let $M_{B}$ be the set of such matching-edges $\left(x_{0}, x_{1}\right)$ i.e., matching-edges that belong to the blossom of such a 7-flower. Note that we do not include the matching-edge $(s, t)$ in $M_{B}$ (unless it belongs to a different 7 -flower where it is in the blossom). Let $T_{B}$ be the set of such vertices $t$, i.e., vertices that belong to a 7-flower and belong to both the stem and the blossom. Set $V_{B}=T_{B} \cup V\left(M_{B}\right)$.

Claim 2. $\left|T_{B}\right| \leq\left|M_{B}\right|$.

Proof. Assign each $t \in T_{B}$ to a matching-edge $\left(x_{0}, x_{1}\right) \in M_{B}$ that is within the same blossom of some 7-flower of $G \backslash V_{C}$. Assume for contradiction that some other vertex $t^{\prime} \in T_{B}$ is also assigned to $\left(x_{0}, x_{1}\right)$. Let $t-s-f$ and $t^{\prime}-s^{\prime}-f^{\prime}$ be the stems of the 7 -flowers containing $t$ and $t^{\prime}$, and note that $s \neq s^{\prime}$ since they are matching-partners of $t \neq t^{\prime}$. This gives an alternating path $f-s-t-x_{0}-x_{1}-t^{\prime}-s^{\prime}-f^{\prime}$, see Figure 2(b). Depending on whether $f=f^{\prime}$ this is a 7-augmenting path or 7-cycle-flower; the former contradicts the choice of $M$ and the latter that $\left(x_{0}, x_{1}\right) \notin M_{C}$.

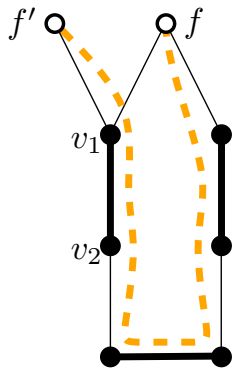

(a)

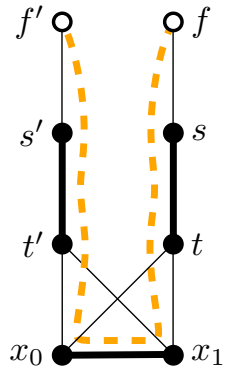

(b)

Figure 2: Augmenting paths found in the proofs of (a) Claim 1 and (b) Claim 2.

The auxiliary graph $H$ For any vertex $v$, let the distance to a free vertex be the number of edges in a shortest alternating path from a free vertex to $v$. Let $D_{k}$ be the vertices of distance $k$ to a free vertex, and observe that there are no edges from $D_{0}$ to $D_{3}$, else there would be a shorter alternating path. We consider these sets as they are found in the graph $G \backslash V_{C} \backslash V_{B}$ 
Observation 1. In graph $G \backslash V_{C} \backslash V_{B}$, there are no matching-edges within $D_{k}$ for $k=1$ and $k=3$, and no edges at all within $D_{k}$ for $k=0$ and $k=2$.

Proof. If there was such an edge $\left(v, v^{\prime}\right)$, then it, together with the alternating paths of length $k$ that lead from free vertices to $v, v^{\prime}$, form a 7 -augmenting path or a 7 -flower.

From now on, we will only study the subgraph $H$ induced by $D_{0} \cup \cdots \cup D_{3}$, noting again that this does not include the vertices in $V_{C} \cup V_{B}$. For ease of referring to them, we rename the vertices of $H$ as follows (see also Figure 3):

- $F_{H}=F \backslash F_{C}=D_{0}$ are the free vertices in $H$.

- $S=D_{1}$ are the vertices adjacent to free vertices in $H$.

- $T_{H}=D_{2}$ are the vertices in $H$ that have matching-partners in $S$ and are not in $S$.

- $U=D_{3}$ are the vertices in $H$ that are adjacent to $T_{H}$ and not in $F \cup S \cup T_{H}$.

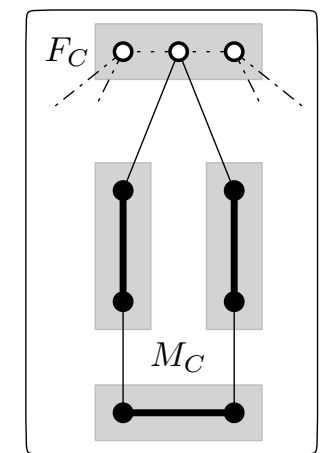

$V_{C}$

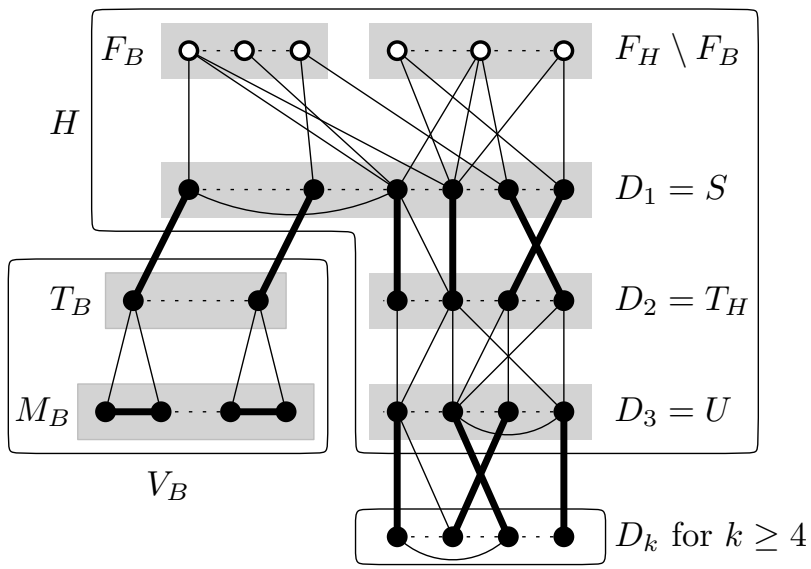

Figure 3: Illustration of the partitioning of edges and vertices. The free vertices $F=F_{C} \cup F_{H}$, the matching edges $M=M_{C} \cup M_{S} \cup M_{U}$, and the remaining vertices $S \cup T_{H} \cup U$ in $H$.

The following shortcuts will be convenient. For any vertex sets $A, B$, an $A$-vertex is a vertex in $A$, an $A$-neighbour is a neighbour of a vertex in $A$, and an $A B$-edge is an edge connecting a vertex in $A$ with a vertex in $B$. Using Observation 1 and the definition of $V_{C}$ (which includes the entire flower) and $V_{B}$ (which includes both ends of the matching-edge) one easily verifies the following:

Observation 2. - There are no matching-edges within $S$ or within $U$.

- There are no edges within $F_{H}$ or within $T_{H}$.

- The matching-partner of an $S$-vertex is in $T_{H} \cup T_{B}$.

- The matching-partner of a U-vertex is not in $H$. 

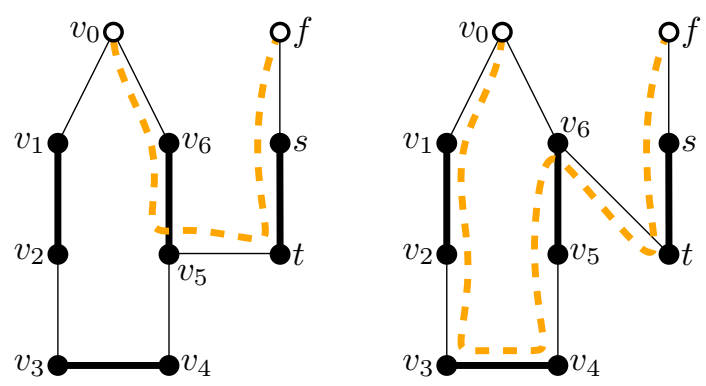

(a)
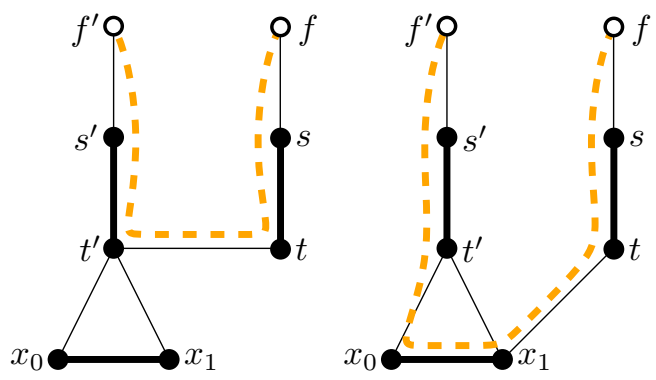

(b)

Figure 4: Augmenting paths found in the proofs of (a) Lemma 1, $t \in T_{H}$ has a neighbour in $V_{C}$. (b) Lemma 1, $t \in T_{H}$ has a neighbour in $V_{B}$.

- All neighbours of an $F_{H}$-vertex belong to $S$ or are not in $H$.

- All neighbours of a $T_{H}$-vertex belong to $S \cup U$ or are not in $H$.

Let $M_{S}$ be the set of matching-edges incident to $S$. Let $M_{U}$ be the matching-edges incident to $U$. Since there are no matching-edges within $S$ or $U$, we have $\left|M_{S}\right|=|S|$ and $|U|=\left|M_{U}\right|$.

We stated earlier that any neighbour of $F_{H}$ is either in $S$ or not in $H$. The latter is actually impossible (though this is non-trivial), and likewise for $T_{H}$.

Lemma 1. No vertex in $F_{H} \cup T_{H}$ has a neighbour in $G$ that is outside $H$.

Proof. First observe that no edge can connect a vertex in $F_{H} \cup T_{H}=D_{0} \cup D_{2}$ with a vertex $z \in D_{k}$ for $k \geq 4$ since $z$ would have been added to $D_{1}=S$ or $D_{3}=U$ instead. So we must only show that no vertex in $F_{H} \cup T_{H}$ has a neighbour in $V_{C} \cup V_{B}$. We show the claim only for $t \in T_{H}$; the proof is similar (and even easier) for $f \in F_{H}$ by replacing the path $t$ - $s$ - $f$ defined below with just $f$.

Figure 4(a) illustrates the following. Fix some $t \in T_{H}$, let $s \in S$ be its matching-partner and let $f \in F_{H}$ be an arbitrary free vertex incident to $s$. Assume for contradiction that $t$ has a neighbour $v_{i}$ in $V_{C}$, so $v_{i}$ belongs to some 7-cycle-flower $v_{0}-v_{1}-\ldots-v_{k}-v_{0}$ where $k \in\{2,4,6\}$ and $v_{0} \in F$. Note that $v_{0} \neq f$ since $v_{0} \in F_{C}$ while $f \in F_{H}$. If $i$ is odd then $f-s-t-v_{i^{-}} \ldots-v_{k}-v_{0}$ is a 9-augmenting path, and if $i$ is even then $f-s-t-v_{i}-v_{i-1}-\ldots-v_{1}-v_{0}$ is a 9-augmenting path; both are impossible.

Now consider some $\left(x_{0}, x_{1}\right) \in M_{B}$ that belongs to a 7 -flower $f^{\prime}-s^{\prime}-t^{\prime}-x_{0}-x_{1}-t^{\prime}-s^{\prime}-f^{\prime}$ where $\left(s^{\prime}, t^{\prime}\right)$ is a matching-edge and $t^{\prime} \in T_{B}$. Note that $t^{\prime} \neq t$ (hence $s^{\prime} \neq s$ ) since $t^{\prime} \in T_{B}$ while $t \in T_{H}$. If $t$ and $t^{\prime}$ are adjacent, then $f-s-t-t^{\prime}-s^{\prime}-f^{\prime}$ is a 5 -augmenting path or a 5 -cycle-flower. If $t$ and $x_{i}$ are adjacent for $i \in\{0,1\}$, then $f-s-t-x_{i}-x_{1-i}-t^{\prime}-s^{\prime}-f^{\prime}$ is a 7 -augmenting path or 7-cycle-flower. See Figure 4(b), There cannot be such augmenting paths, and no such cycle-flowers either since $t \notin T_{C}$.

In particular, if a vertex in $F_{H} \cup T_{H}$ had degree $d$ in $G$, then it also has degree $d$ in $H$; this will be important in obtaining matching-bounds below. 
Minimum degree 3 With this, we can prove our first matching-bound. We need the following lemma shown by Biedl and Wittnebel:

Lemma 2 ([5]). Let $G$ be a simple 1-planar graph. Let $A$ be a non-empty independent set of $G$ where all vertices in $A$ have degree 3 or more in $G$. Let $A_{d}$ be the vertices of degree $d$ in $A$. Then

$$
2\left|A_{3}\right|+\sum_{d>3}(3 d-6)\left|A_{d}\right| \leq 12|V \backslash A|-24 .
$$

Lemma 3. We have (i) $\left|F_{H}\right| \leq 6|S|-12$ and (ii) $\left|F_{H}\right|+\left|T_{H}\right| \leq 6|S|+6|U|-12$.

Proof. Consider first the subgraph of $H$ induced by $F_{H}$ and $S$. By Observation 2 and Lemma 1 any vertex in $F_{H}$ has degree at least 3 in this subgraph, and they form an independent set. Consider the inequality of Lemma 2. Any vertex in $F_{H}$ contributes at least 2 units to the LHS while the RHS is $12|S|-24$. This proves Claim (i) after dividing.

Now consider the full graph $H$. By Observation 2 and Lemma 1 any vertex in $F_{H} \cup T_{H}$ has degree at least 3 in $H$, and they form an independent set. Claim (ii) now follows from Lemma 2 as above.

Corollary 1. If the minimum degree is 3, then $|M| \geq \frac{7}{50}(n+12)$.

Proof. Adding Lemma 3(ii) six times to Lemma 3(i) gives

$$
7\left|F_{H}\right|+6\left|T_{H}\right| \leq 42|S|+36|U|-84 \leq 42\left|M_{S}\right|+36\left|M_{U}\right|-84 .
$$

Adding Claim 1 seven times and Claim 2 six times gives

$$
7\left|F_{C}\right|+7\left|F_{H}\right|+6\left|T_{B}\right|+6\left|T_{H}\right| \leq 42\left|M_{S}\right|+36\left|M_{U}\right|+7\left|M_{C}\right|+6\left|M_{B}\right|-84 .
$$

Since $|S|=\left|M_{S}\right|=\left|T_{H}\right|+\left|T_{B}\right|$, this simplifies to

$$
7|F|=7\left|F_{H}\right|+7\left|F_{C}\right| \leq 36\left|M_{S}\right|+36\left|M_{U}\right|+7\left|M_{C}\right|+6\left|M_{B}\right|-84 \leq 36|M|-84 .
$$

Therefore $2|M|=n-|F| \geq n+12-\frac{36}{7}|M|$ which gives the bound after rearranging.

It is worth pointing out that this result (as well as Theorem 2 below) does not use 1planarity of the graph except when using the bound in Lemma 2. Hence, similar bounds could be proved for any graph class where the size of independent sets can be upper-bounded relative to its minimum degree.

Doing the improvement from $\frac{7}{50}$ to $\frac{1}{7}$ will be done by improving the bound on $\left|F_{H}\right|+\left|T_{H}\right|$ slightly. By modifying $H$ and its 1-planar drawing and studying a resulting 1-planar bipartite graph $J$, we will show the following in Section 5 :

Lemma 4. $\left|F_{H}\right|+\left|T_{H}\right| \leq 6|S|+5|U|-12$.

This then gives our main result: 
Theorem 1. Let $G$ be a 1-planar graph with minimum degree 3, and let $M$ be a matching in $G$ that has no augmenting path of length 9 or less. Then $|M| \geq \frac{n+12}{7}$.

Proof. Using $|S|=\left|M_{S}\right|$ and $|U|=\left|M_{U}\right|$ we have

$$
\begin{aligned}
\left|F_{H}\right|+\left|T_{H}\right| & \leq 6\left|M_{S}\right|+5\left|M_{U}\right|-12 & & \text { from Lemma 4 } \\
\left|F_{C}\right| & \leq\left|M_{C}\right| & & \text { from Claim 1 } \\
\left|T_{B}\right| & \leq\left|M_{B}\right| & & \text { from Claim 2, }
\end{aligned}
$$

Since $\left|T_{H}\right|+\left|T_{B}\right|=\left|M_{S}\right|$ this gives $|F|+\left|M_{S}\right| \leq\left|M_{C}\right|+\left|M_{B}\right|+6\left|M_{S}\right|+5\left|M_{U}\right|-12$, therefore $|F| \leq 5|M|-12$. This implies $2|M|=n-|F| \geq n-5|M|+12$ or $7|M| \geq n+12$.

Higher minimum degree Since the bound for independent sets in 1-planar graphs gets smaller when the minimum degree is larger, we can prove better matching-bounds for higher minimum degree.

Lemma 5. If the minimum degree is $\delta>3$, then

$$
\text { (i) }\left|F_{H}\right| \leq \frac{4}{\delta-2}(|S|-2) \quad \text { and } \quad(i i)\left|F_{H}\right|+\left|T_{H}\right| \leq \frac{4}{\delta-2}(|S|+|U|-2) \text {. }
$$

Proof. As in Lemma 3, consider the subgraph of $H$ induced by $F_{H}$ and $S$. Any $f \in F_{H}$ has degree $\delta$ or more and contributes at least $3 \delta-6$ units to the LHS of the inequality in Lemma 2. The RHS is $12|S|-24$. This proves Claim (i) after dividing. Claim (ii) is proved the same way using the full graph $H$.

Theorem 2. Let $G$ be a 1-planar graph with minimum degree $\delta$. Let $M$ be any matching in $G$ without 9-augmenting path. Then

- $|M| \geq \frac{3}{10}(n+12)$ for $\delta=4$,

- $|M| \geq \frac{1}{3}(n+12)$ for $\delta \geq 5$.

Proof. Set $c=\frac{4}{\delta-2}$, so $\left|F_{H}\right| \leq c(|S|-12)$ and $\left|F_{H}\right|+\left|T_{H}\right| \leq c(|S|+|U|-12)$. Taking the former inequality once and adding the latter one $c$ times gives

$$
(c+1)\left|F_{H}\right|+c\left|T_{H}\right| \leq\left(c^{2}+c\right)|S|+c^{2}|U|-(c+1) 12=\left(c^{2}+c\right)\left|M_{S}\right|+c^{2}\left|M_{U}\right|-(c+1) 12 .
$$

Adding Claim 1$] c+1$ times and Claim $2 c$ times gives

$$
(c+1)\left(\left|F_{C}\right|+\left|F_{H}\right|\right)+c\left(\left|T_{B}\right|+\left|T_{H}\right|\right) \leq\left(c^{2}+c\right)\left|M_{S}\right|+c^{2}\left|M_{U}\right|+(c+1)\left|M_{C}\right|+c\left|M_{B}\right|-(c+1) 12 .
$$

For $\delta=4$ we have $c=2$, and with $\left|T_{B}\right|+\left|T_{H}\right|=\left|M_{S}\right|$ Equation 1 simplifies to

$$
3|F| \leq 4\left|M_{S}\right|+4\left|M_{U}\right|+3\left|M_{C}\right|+2\left|M_{B}\right|-36 \leq 4|M|-36 .
$$

Therefore $2|M|=n-|F| \geq n+12-\frac{4}{3}|M|$. For $\delta \geq 5$ we have $c^{2}<c+1$ and so can only simplify Equation 1 to

$$
(c+1)\left(\left|F_{C}\right|+\left|F_{H}\right|\right) \leq(c+1)|M|-(c+1) 12
$$

hence $2|M|=n-|F| \geq n+12-|M|$. The bounds follow after rearranging. 
For $\delta=4,5$ these are close to the bounds of $\frac{1}{3}(n+4)$ (for $\left.\delta=4\right)$ and $\frac{1}{5}(2 n+3)$ (for $\delta=5$ ) that we know to be the tight lower bounds on the maximum matching size [5]. Unfortunately we do not know how improve Theorem 2 for $\delta>3$; the techniques of Section 5 do not work for higher minimum degree since we will use another inequality (Observation 4(i)) that is not strong enough to achieve the bound for higher degrees, and not easily improved.

The case $\delta \geq 6$ is also interesting. Here one would hope for even larger matching-bounds. Unfortunately, the bottleneck in our analysis is our treatment of flowers of length 3. Here we remove one free vertex and one matching-edge, which can at best lead to a bound of $|M| \geq \frac{1}{3}(n+O(1))$. So a further improvement of the bound for minimum degree $\delta \geq 6$ would require treating short flowers differently.

\subsection{Stopping earlier?}

Currently we remove all augmenting paths up to length 9 before returning the matching. Naturally one wonders whether one could stop earlier?

It is possible to show that it would suffice to remove only 7 -augmenting paths. Inspecting the analysis, one sees that the absence of augmenting paths of length exactly 9 is used only once: In the proof of Lemma 1, we use it to argue that a vertex $t \in T_{H}$ is not adjacent to a 7 -cycle flower. Digging further, one can verify that 7 -cycle-flowers need to be removed only to avoid matching-edges within $U$. It turns out that one can deal with matching-edges within $U$ directly, by arguing that at most three vertices in $T_{H}$ can have an endpoint at such an edge (else there is a 7-augmenting path), and removing these vertices and matching-edges and accounting for them directly. The details are not difficult but tedious and require even more notation; we will not give them.

On the other hand, it is not enough to remove only 3 -augmenting paths. Figure 5 shows an example of a matching in a 1-planar graph that has no 3-augmenting paths, but only size $\frac{n+12}{8}$. We can show that this is as bad as it can get.

Theorem 3. Let $G$ be a 1-planar graph with minimum degree 3 and let $M$ be a matching without 3-augmenting paths. Then $|M| \geq \frac{n+12}{8}$.

Proof. The proof is very similar to the one of Theorem 2 in [12] except that we use Lemma 2 rather than the edge-bound for planar bipartite graphs. We repeat it here for completeness, mimicking their notation. Let $M_{c}$ be all those matching-edges $(x, y)$ for which some free vertex $f \in F$ is adjacent to both $x$ and $y$, and let $F_{c}$ be all such free vertices. Vertex $f$ is necessarily the only $F$-neighbour of $x$ and $y$, else there would be a 3 -augmenting path. Hence $\left|F_{c}\right| \leq\left|M_{c}\right|$.

Let $M_{o}$ and $F_{o}$ be the remaining matching-edges and free vertices. For each edge $(x, y)$ in $M_{o}$, at most one of the ends can have $F$-neighbours, else $(x, y)$ would be in $M_{c}$ or there would be a 3 -augmenting path. Let $S$ be the ends of edges in $M_{o}$ that have $F$-neighbours, and let $G^{\prime}$ be the auxiliary graph induced by $F$ and $S$. Since $F$ is an independent set, we have $\left|F_{o}\right| \leq 6|S|-12 \leq 6\left|M_{o}\right|-12$ by Lemma 2 .

Putting both together, $2|M|=n-|F| \geq n+12-\left|M_{c}\right|-6\left|M_{o}\right| \geq n+12-6|M|$ and the bound follows after rearranging. 


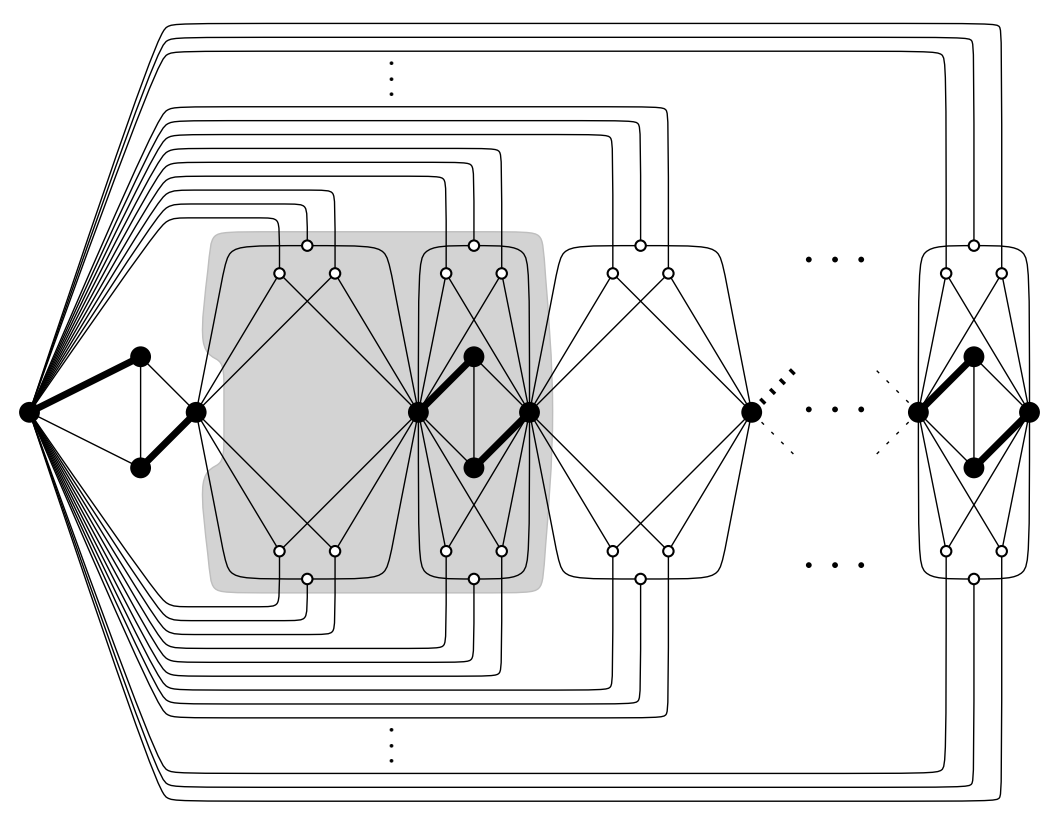

Figure 5: A graph with a matching marked in thick edges of size $\frac{n+12}{8}$. No 3-augmenting path exists for the chosen matching, but there are 5-augmenting paths. The gray area marks an example of 16 vertices such that only 2 matching edges exist. Repeating this configuration gives the example for arbitrary $n$.

\section{Proof of Lemma 4}

In this section, we prove Lemma 4 , i.e., we show that

$$
\left|F_{H}\right|+\left|T_{H}\right| \leq 6|S|+5|U|-12 \text {. }
$$

The following proof does not quite work, but puts us in the right direction. Consider the graph $H$ defined earlier. For any $t \in T_{H}$ that has $U$-neighbours, contract $t$ into one of its $U$-neighbours. In the resulting graph all $F_{H}$-vertices and the remaining $T_{H}$-vertices have three $S$-neighbours. If a $U$-vertex had $d T_{H}$-vertices contracted into it, then it now has degree at least $d$ (because the matching-partners of the $T_{H}$-vertices are distinct). Also, all vertices not in $S$ form an independent set. If the resulting graph is 1-planar, we could hence use Lemma 2 to bound $\left|F_{H}\right|+\left|T_{H}\right|$ as sufficiently small.

Unfortunately, 1-planar graphs are not closed under contraction in general; we can only contract along uncrossed edges. So we need to proceed more carefully, the following is an outline. We first assign $T_{H^{-}}$-vertices to a carefully chosen $U$-neighbour $\left(T_{H^{-}}\right.$-verties that have no $U$-neighbours will not be contracted). Next eliminate $U$-vertices with few assigned $T_{H^{-}}$ vertices; these can be accounted for easily. In the resulting drawing $I$, we contract each remaining $T_{H}$-vertex that has $U$-neighbours along an uncrossed edges. Unfortunately we cannot always do a contraction along the matching-edge or the assignment-edge, which will make it more difficult to argue that a vertex in $U$ retain sufficiently high degrees. Letting $J$ be the resulting final drawing, we then apply Lemma2 (in a stronger form) to the bipartite graph 
$J$ to prove Lemma 4. We phrase the various procedures below as if they were algorithms, but remind the reader that they are only used for the analysis and not needed for finding the matching.

The super-graph $H^{+}$. Fix an arbitrary 1-planar drawing of $H$; from now on we use $H$ (as well as the graphs $H^{+}, I, J, J^{-}$derived from it) to mean both the graph and the 1-planar drawing that comes with it, and which will not be changed unless stated explicitly.

We obtain a 1-planar drawing $H^{+}$by changing $H$ in two ways. First, delete all edges of $H$ that are within $S \cup U$. Second, add/re-route kite-edge as follows. Let $(t, x)$ by a potential kite-edge of some crossing $c$ of $H$ with $t \in T_{H}$ and $x \in U \cup S$. If $(t, x)$ does not yet exist in $H$, then add it. If $(t, x)$ exists already in $H$ and is crossed, then re-route $(t, x)$ as the kite-edge so that it becomes uncrossed. If $(t, x)$ exists as uncrossed edge in $H$ already, then do not insert it (so that $\mathrm{H}^{+}$stays simple). However, if crossing $c$ involves the matching-edge at $t$, then re-route $(t, x)$ (if needed) to be at crossing $c$ rather than elsewhere. Repeat for all such potential kite-edges $(t, x)$. See also Figure6.

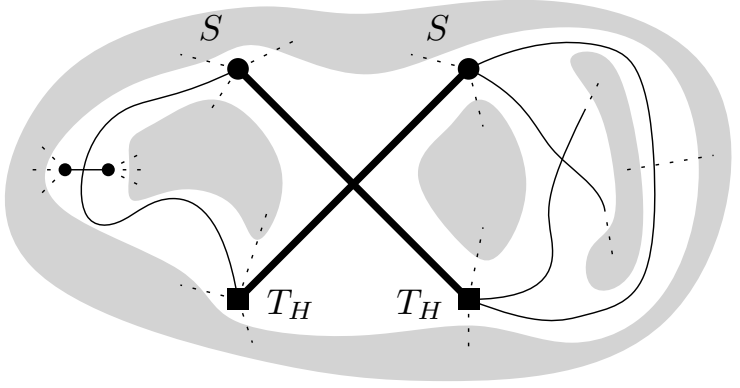

(a)

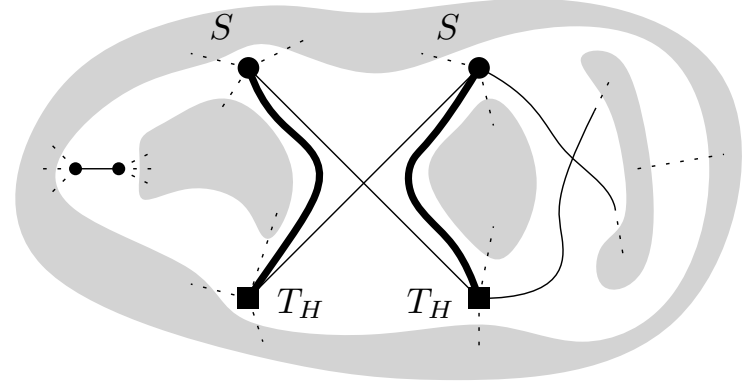

(b)

Figure 6: Re-routing kite-edges that are crossed or not routed near the matching edge, and trading matching-partners. The gray areas symbolize other parts of the graph. Dotted edges indicate possible connections.

We also trade matching-partners as follows. Assume that $H^{+}$contains two matching edges $(s, t)$ and $\left(s^{\prime}, t^{\prime}\right)$ (with $s, s^{\prime} \in S$ and $t, t^{\prime} \in T_{H}$ ) that cross each other. We inserted (or re-drew) kite-edges $\left(s, t^{\prime}\right)$ and $\left(s^{\prime}, t\right)$ at this crossing. We now remove edges $(s, t)$ and $\left(s^{\prime}, t^{\prime}\right)$ from the matching and declare $\left(s, t^{\prime}\right)$ and $\left(s^{\prime}, t\right)$ to be matching-edges instead, noting that these are uncrossed. This exchange would not necessarily have been possible in $G$ where these kite-edges need not exist, but we do it here only for the purpose of explaining how to transform $T_{H}$-vertices and do not actually change the returned matching. From now on, "matching-edge" and "matching-partner" refers to the status after this trading. Note that all vertices in $T_{H}$ remain matched.

We summarize the properties of $H^{+}$for future reference:

Observation 3. $\mathrm{H}^{+}$is a simple graph with a fixed 1-planar drawing that satisfies the following. 
(a) Any edge has exactly one endpoint in $S \cup U$.

(b) Vertices in $F_{H}$ have degree 3 or more and all their neighbours are in $S$. Vertices in $T_{H}$ have degree 3 or more and all their neighbours are in $S \cup U$.

(c) If $(x, t)$ is a potential kite-edge at crossing c for some $x \in S \cup U$ and $t \in T_{H}$, then $(x, t)$ exists as an uncrossed edge in $H^{+}$. If $c$ involves the matching-edge at $t$, then $(x, t)$ is routed at crossing $c$.

(d) No two crossing matching-edges cross each other.

Proof. To show (a), observe that in $H$ every edge has at least one endpoint in $S \cup U$ by Observation 2. Added kite-edges also have an endpoint in $S \cup U$, There is exactly one such endpoint since we deleted edges within $S \cup U$. (b) held in $H$ by Observation 2 and also holds for added kite-edges. (c) and (d) hold by construction of $\mathrm{H}^{+}$and since we traded matching-partners.

Assignment to $U$-vertices. We assign vertices in $T_{H}$ to $U$-neighbours with the following AssignmentAlgorithm. For each $t \in T_{H}$ :

- If $t$ has an uncrossed edge to a $U$-neighbour:

- Add $t$ to a set $T_{\mu}$. (As we will see, vertices in $T_{\mu}$ use their matching-edges during the transformation explained later, hence the " $\mu$ ".)

- If the matching-edge $(t, s)$ is crossed by an edge $(y, u)$ with $u \in U$, then assign $t$ to this $u$ (noting that edge $(t, u)$ exists as uncrossed kite-edge at this crossing).

- Otherwise assign $t$ to an arbitrary $U$-neighbour $u$ for which edge $(t, u)$ is uncrossed.

- Otherwise, if $t$ has at least three $S$-neighbours, add $t$ to $T_{\sigma}$. (The " $\sigma$ " reminds that there are sufficiently many $S$-neighbours that we do not need to transform these vertices.)

- Otherwise, all edges to $U$-neighbours are crossed. Add $t$ to a set $T_{\rho}$ (" $\rho$ " stands for "remaining"). Assign $t$ to an arbitrary $U$-neighbour.

If $t \in T_{H}$ has been assigned to $u \in U$, then we call $(t, u)$ the assignment-edge of $t$. Let $U^{d}$ be the set of all those vertices $u \in U$ that had $d$ incident assignment-edges. It will be helpful to assign as many $T$-vertices as possible to $\bigcup_{d \leq 5} U^{d}$; we therefore do the following re-assignment: If $t \in T_{H}$ is assigned to a vertex $u \notin \bigcup_{d \leq 5} U^{d}$, but $t$ has a neighbour $u^{\prime} \in U^{0} \cup \cdots \cup U^{4}$, then re-assign $t$ to $u^{\prime}$. Note that this changes the sets $U^{d}$, but it can only increase $\bigcup_{d \leq 5} U^{d}$ since we never assign another vertex of $T_{H}$ to a vertex in $U^{5}$. Repeat (with the new sets $U^{0}, \ldots, U^{5}$ ) until no more re-assignments are possible.

Let $T^{d}$ be the vertices in $T_{H} \backslash T_{\sigma}$ that are assigned to a vertex in $U^{d}$. So $\left|T^{d}\right|=d\left|U^{d}\right|$, and with our choice of assignment-edge, we have the following trivial (but crucial) properties:

Observation 4. (i) $\left|T_{0}\right|=0$ and $\sum_{d=1}^{5}\left|T^{d}\right| \leq 5 \sum_{d=0}^{5}\left|U^{d}\right|$. 
(ii) If $t \in T^{d}$ with $d \geq 6$ has an uncrossed edge to a $U$-neighbour, then $t$ belongs to $T_{\mu}$.

(iii) If $t \in T^{d}$ with $d \geq 6$ has a neighbour $u$ in $\bigcup_{j \leq 5} U^{j}$, then $u \in U^{5}$.

In particular, using (i) the vertices in $\bigcup_{d \leq 5} T^{d}$ can be accounted for directly by the matching edges incident to $\bigcup_{d \leq 5} U^{d}$. We only need to bound the remaining $T_{H}$-vertices, which are those in $T_{\sigma}$ as well as those in $\bigcup_{d \geq 6} T^{d}$.

The easy transformations. We now explain the contractions mentioned in the outline. However, we prefer to view them as "transformations" (consisting of deleting one vertex $t$ and inserting one edge $(s, u)$ between neighbours of $t$ ) since the route used for the new edge must be described carefully. We first do the "easy" transformations where $s$ can be chosen to be the matching-partner of $t$. Let $I$ be the 1-planar drawing obtained from $H^{+}$as follows:

- Delete all vertices in $\bigcup_{d \leq 5}\left(U^{d} \cup T^{d}\right)$.

- For any remaining vertex $t \in T_{H}$, delete all edges to $U$-neighbours except the assignmentedge (if any).

- While there exists a vertex $t \in T_{H} \backslash T_{\sigma}$ for which either the assignment-edge $(t, u)$ or the matching-edge $(s, t)$ is uncrossed:

- Delete $t$

- If the path $s-t$ - $u$ had a crossing $c$ with an edge $(x, y)$ that has an endpoint in $\{s, u\}$ : Insert $(s, u)$ as a kite-edge of crossing $c$. We call this a $\kappa$-transformation (the " $\kappa$ " reminds of "kite").

- Otherwise insert $(s, u)$, drawing it along path $s-t$ - $u$, and observe that this has at most one crossing and gives a good drawing. We call this a $\pi$-transformation (the " $\pi$ " reminds of "path").

Figure 7 (a-c) shows an example of this transformation algorithm. Note that the transformation will remove all vertices in $T_{\mu}$, and it may also remove some vertices of $T_{\rho}$, since their matching-edges and/or assignment-edges may become uncrossed as other vertices are deleted. Let $T_{\mu}^{\prime}$ be all those vertices that were removed and let $T_{\rho}^{\prime} \subseteq T_{\rho}$ be the vertices $T_{H} \backslash \bigcup_{d \leq 5} T^{d} \backslash T_{\sigma} \backslash T_{\mu}^{\prime}$, i.e., all those vertices that still need to be removed.

Observation 5. Drawing $I$ is simple and good and any vertex $u \in U^{d}$ with $d \geq 6$ has degree $d$ in $I$.

Proof. Vertex $u$ had $d$ incident assignment-edges, say to $t_{1}, \ldots, t_{d}$. For each $t_{i}$, if $t_{i} \in T_{\rho}^{\prime}$ then it remains a neighbour in $I$. If $t_{i} \in T_{\mu}^{\prime}$, then edge $\left(t_{i}, u\right)$ in $H^{+}$has been replaced by edge $\left(s_{i}, u\right)$ in $I$, where $s_{i}$ is the matching-partner of $t_{i}$. This does not create multiple edges since we deleted all $S U$-edges that existed previously in $H^{+}$, and since no $s_{i}$ is matched to two vertices. The drawing is good since we use an $\kappa$-transformation if drawing along the path $s_{i}-t_{i}-u$ would have violated goodness. 


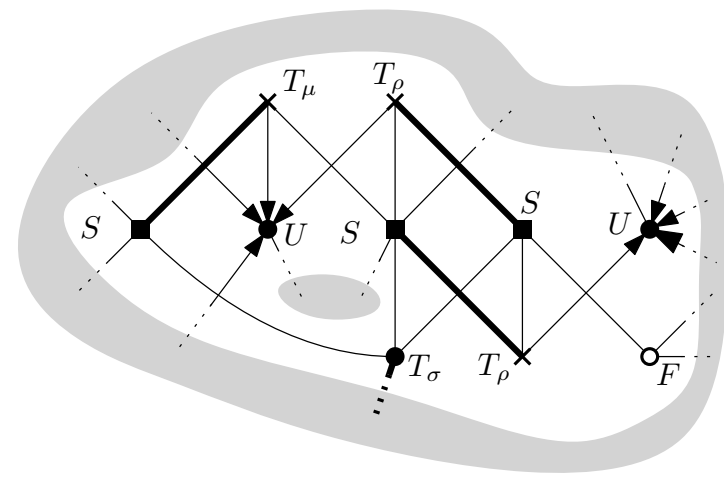

(a)

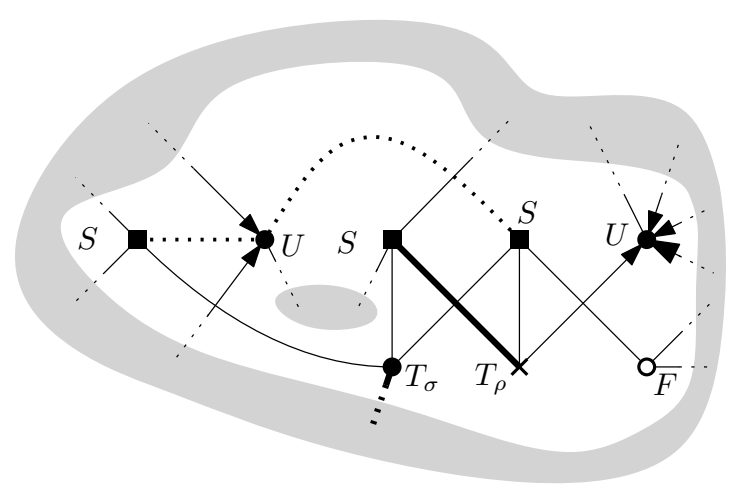

(c)

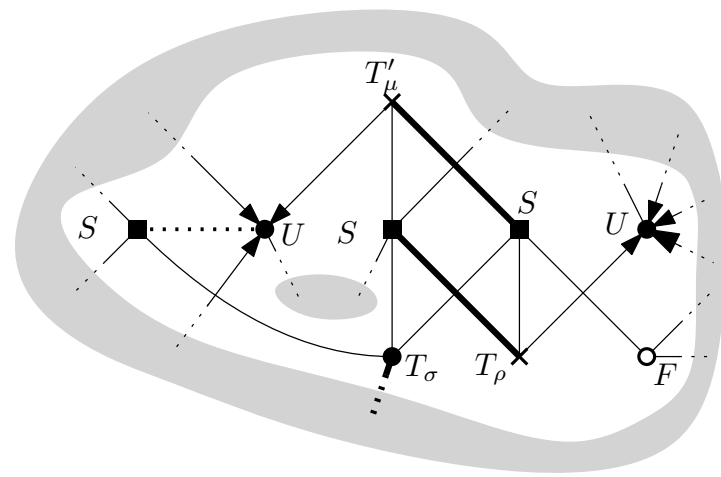

(b)

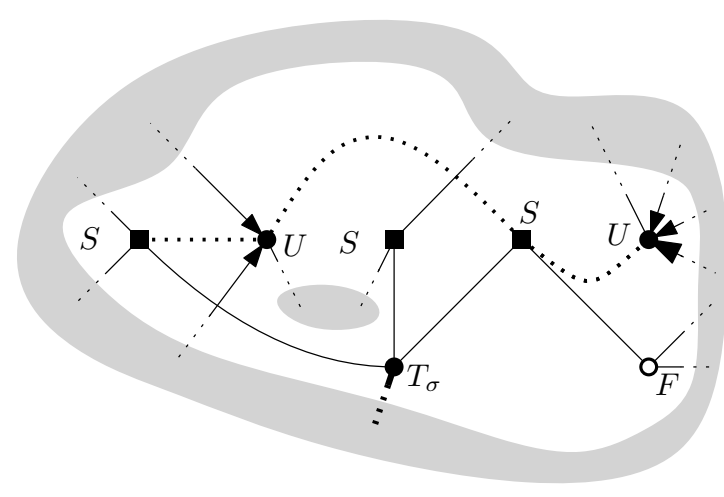

(d)

Figure 7: (a) A part of a graph $\mathrm{H}^{+}$; arrows indicate assignments. Vertices are labeled with the sets they belong to. Gray areas symbolize the remaining graph and its incident dotted edges are possible connections. (b) An $\kappa$-transformation: The new edge gets routed as kiteedge. This causes one vertex in $T_{\rho}$ to move to $T_{\mu}^{\prime}$. (c) A $\pi$-transformation: The new edge gets routed along the path through the eliminated vertex. (d) A $\rho$-transformation.

We call a region a kite-region if it is bounded by two half-edges at a crossing $c$ and an uncrossed edge (necessarily a kite-edge of $c$ ).

Claim 3. Let $(s, u)$ be an uncrossed edge in $I$ with $s \in S$ and $u \in U$. Then at most one of the two regions incident to $(s, u)$ in $I$ is a kite-region.

Proof. Since we deleted $S U$-edges of $\mathrm{H}^{+}$as first step of the transformation, edge $(s, u)$ was added during the transformation, say at vertex $t \in T_{\mu}^{\prime}$ for which $(s, t)$ was a matching-edge. If this was an $\kappa$-transformation, then $(s, u)$ is drawn as kite-edge of some crossing $c$ that involved either $(s, t)$ or $(t, u)$. But the edges $s$ - $t$ - $u$ are removed from the drawing, so $c$ no longer exists as crossing in $I$. So in $I$, the region incident to $(s, u)$ where $c$ used to be is not a kite-region. See Figure 8(a).

Now assume that $t$ underwent a $\pi$-transformation, and assume for contradiction that there are two crossings $c_{1}, c_{2}$ at which the inserted edge $(s, u)$ bounds kite-regions. Therefore 


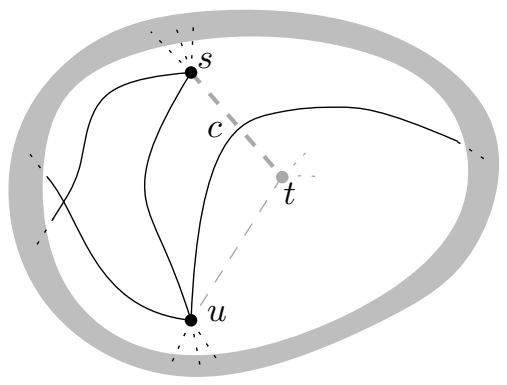

(a)

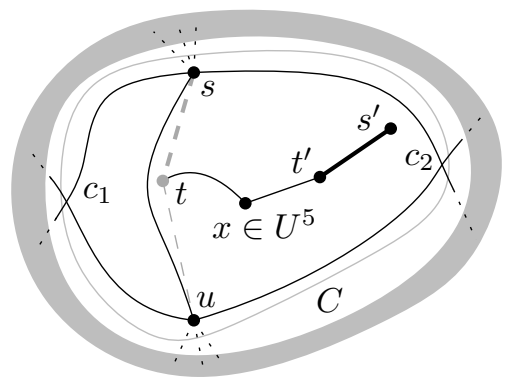

(b)

Figure 8: Illustration of the situation in Claim 3 if $t$ underwent (a) a $\kappa$-transoformation, and (b) a $\pi$-transformation and $x \in U^{5}$.

in drawing $I$ we have the curve $C:=s-c_{1}-u-c_{2}-s$ that contains $(s, u)$ on one side (say the inside) and everything else on the other side. Figure 8(b) gives an illustration. In drawing $H^{+}$, curve $C$ also existed, and contained $t$ on the inside. Since no crossed edge can be crossed again, any neighbours of $t$ in $H^{+}$lies on or inside $C$. There are at least three neighbours of $t$ by Observation 3 . Two of these are $s$ and $u$, but there exists a third neighbour $x \in S \cup U$ of $t$ inside $C$. If $x \in S$ or $x \in U^{d}$ for $d \geq 6$ then $x$ also exists in $I$ and lies inside $C$, contradicting that we had only the kite-regions inside $C$ in $I$. So $x \in \bigcup_{d<5} U^{d}$, which by Observation 4 means $x \in U^{5}$. So at least one other $T_{H^{-}}$vertex $t^{\prime}$ is assigned to $x$. Let $s^{\prime}$ be the matching-partner of $t^{\prime}$, so $s^{\prime} \neq s$. Path $x-t^{\prime}-s^{\prime}$ is disjoint from $s, u$ and therefore must also lie inside $C$ in $H^{+}$. Since $s^{\prime} \in S$ remains in $I$, this is again a contradiction.

The vertices in $T_{\rho}^{\prime}$. The vertices in $T_{\rho}^{\prime}$ that could not be removed yet have a special structure in their neighbourhood that is illustrated in Figure 9(a).

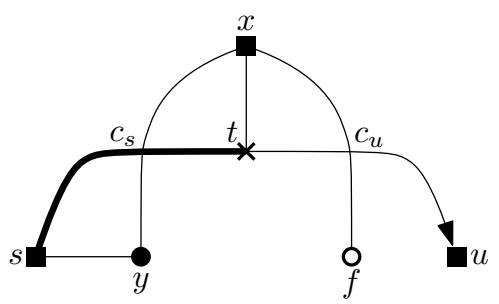

(a)

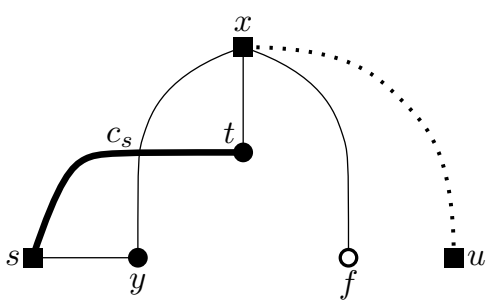

(b)

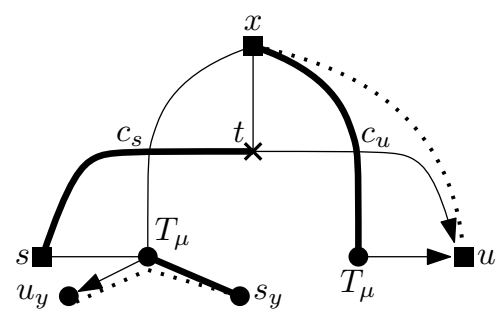

(c)

Figure 9: (a) The structure at a vertex $t \in T_{\rho}^{\prime}$. (b) Doing a $\rho$-transformation at $t$. (c) If $y \in T_{\mu}$ or $f \in T_{\mu}$, then $c_{s}$ respectively $c_{u}$ would not exist in $I$.

Lemma 6. Fix a vertex $t \in T_{\rho}^{\prime}$. Let $(t, s)$ and $(t, u)$ be its matching-edge and assignmentedge. Then in drawing $I$ :

(a) The matching-edge $(t, s)$ is crossed by some edge $(x, y)$ with $x \in S$. 
(b) The assignment-edge $(t, u)$ is crossed by some $\left(x^{\prime}, f\right)$ with $x^{\prime} \in S$.

(c) $x=x^{\prime}$ and the edge $(t, x)$ exists as uncrossed edge.

(d) $y \in F_{H} \cup T_{\sigma}$.

(e) $f \in F_{H}$.

Proof. We know that $(t, s)$ and $(t, u)$ are both crossed in $I$ (else $t$ would have been transformed). Since we never add crossings, they were also crossed in $H^{+}$, say $(t, s)$ was crossed by some edge $(x, y)$ at $c_{s}$ and $(t, u)$ was crossed by some $\left(x^{\prime}, f\right)$ at $c_{u}$. We first show that (a-e) hold in $H^{+}$, i.e., for these vertices $x, x^{\prime}, y, f$. By Observation 3(a), up to renaming $x, x^{\prime} \in S \cup U$. By Observation 3(c), edges $(t, x)$ and $\left(t, x^{\prime}\right)$ exist as uncrossed edges. This implies $x, x^{\prime} \in S$ because otherwise $t$ would have an uncrossed edge to a $U$-neighbour, contradicting its crossed assignment-edge $(t, u)$. This proves (a) and (b).

Since edge $(t, s)$ is crossed while $(t, x)$ and $\left(t, x^{\prime}\right)$ are uncrossed, we know $x \neq s \neq x^{\prime}$. If $x \neq x^{\prime}$ then $t$ has three $S$-neighbours and would belong to $T_{\sigma}$ rather than $T_{\rho}$. So $x=x^{\prime}$, which proves (c).

To prove (d), recall that by Observation 3(a) and $x \in S$ we know $y \in T_{H} \cup F_{H}$. Assume that $y \in T_{H}$ (else (d) holds). Then $y$ has the $S$-neighbours $s$ and $x$ since we added kite-edges. Its matching-partner is not $s$ (since $(s, t)$ is a matching-edge) and also not $x$ (else $(s, t)$ and $(x, y)$ would cross each other). So its matching-partner is a third $S$-neighbour $s_{y}$. Since $y$ has three $S$-neighbours, we hence either add it to $T_{\mu}$ or to $T_{\sigma}$. But we cannot have $y \in T_{\mu}$, else it would have been transformed and replaced by edge $\left(u_{y}, s_{y}\right)$ for some $u_{y} \in U$, see Figure 9(c), By $s_{y} \neq x$ this eliminates crossing $c_{s}$, contradicting that $(t, s)$ remains crossed in $I$. So $(\mathrm{d})$ holds.

Proving (e) is similar. By $x \in S$ we know $f \in T_{H} \cup F_{H}$. Assume for contradiction that $f \in T_{H}$. This implies $f \in T_{\mu}$ since $(f, u)$ is a potential kite-edge, hence exists uncrossed in $H^{+}$. So the transformations delete $f$ and insert edge $\left(u_{f}, s_{f}\right)$ where $s_{f}$ and $u_{f}$ are the matching-partner and assigned $U$-neighbour. If $s_{f} \neq x$, then this will eliminate crossing $c_{u}$. If $s_{f}=x$, then our choice of assignment-edges ensured that $u_{f}=u$ and the $\kappa$-transformation inserts $\left(s_{f}, u_{f}\right)=(x, u)$ as kite-edge of $c_{u}$. See Figure 9(c). So either way $(t, u)$ is no longer crossed in $I$, a contradiction and (e) holds.

So we have proved (a-e) for the edges that crossed $(t, s)$ and $(t, u)$ in $H^{+}$. But since their endpoints belong to $S \cup U \cup F_{H} \cup T_{\sigma}$, none of the transformations affect these edges, so the same situation holds in $I$.

We now finish our transformations as follows:

- For any $t \in T_{\rho}^{\prime}$, let the assignment-edge be $(t, u)$, and let $(x, f)$ be the edge that crosses it at $c_{u}$. Delete $t$ and insert $(x, u)$ as kite-edge of $c_{u}$. Insert this edge even if it existed already in $I$. We call this a $\rho$-transformation.

Observe that the only edge that is made uncrossed by a $\rho$-transformation is $(x, f)$. Neither of its endpoints belongs to $T_{\rho}^{\prime}$, so no $\rho$-transformation affects the status of crossed edges at 
some other vertex $t^{\prime} \in T_{\rho}^{\prime}$. Consequently, Lemma 6 continues to hold for all drawings during all $\rho$-transformations. Let $J$ be the final drawing. Observe that $J$ is specfically allowed to have multi-edges (but no loops). It may even have an empty lens (two uncrossed copies of an edge that bound a region). But it does not have an empty $\Theta$, i.e., three uncrossed copies of an edge such that two of the three areas between them are regions of the drawing.

Claim 4. If $J$ has multiple copies of some edge $(x, u)$, then all but at most one copy are uncrossed, and $J$ has no empty $\Theta$.

Proof. Since $I$ is simple, a second copy of $(x, u)$ can be inserted only during a $\rho$-transformation. This adds $(x, u)$ as an uncrossed kite-edge, so only the copy of $(x, u)$ that may have existed in $I$ can be crossed.

Now assume for contradiction that $(x, u)$ exists in three uncrossed copies $e_{1}, e_{2}, e_{3}$ that bound an empty $\Theta$, say the regions bounded by $e_{1}, e_{2}$ and $e_{2}, e_{3}$ are empty lenses. We necessarily have $x \in S$ and $u \in U$, since no other edges are inserted in $J \backslash I$. Since $I$ is simple, at least two of $e_{1}, e_{2}, e_{3}$ were inserted with $\rho$-transformations.

A copy of $(x, u)$ is inserted during a $\rho$-transformation only if it was the kite-edge of some crossing $c_{u}$ between edge $(x, f)$ (for some $f \in F_{H}$ ) and edge $(t, u)$ (where $t \in T_{\rho}^{\prime}$ is being transformed). Edge $(x, f)$ becomes consecutive with the newly inserted copy of $(x, u)$, and both are uncrossed, which means that one region incident to this copy of $(x, u)$ is not an empty lens in $J$. Therefore $e_{2}$ cannot have been inserted with $\rho$-transformations.

The only remaining possibility is that $e_{2}$ already existed in $I$ while $e_{1}$ and $e_{3}$ were inserted with $\rho$-transformations. But then both regions incident to $e_{2}$ in $I$ must have been kite-regions, contradicting Claim 3 .

Crossing-weighted degrees. For any vertex $v$, let the crossing-weighted degree of $v$ be the degree plus the number of incident uncrossed edges. Let $J^{-}$be the drawing obtained from $J$ by deleting one edge from each empty lens.

Observation 6. For any vertex $u \in U^{d}$ with $d \geq 6$, the crossing-weighted degree of $u$ in $J^{-}$ is at least $d$ and the degree is at least 3.

Proof. We know that $u$ had degree $d$ in $I$, and with the same argument it has degree $d$ in $J$. Assume $u$ was incident to $k$ empty lenses in $J$. Since there are no empty $\Theta$ 's, edges in empty lenses come in pairs; in particular $k \leq d / 2$ and we delete $k$ edges. Then the degree of $u$ in $J^{-}$is $d-k \geq d / 2=3$. The $k$ edges that remain from the empty lenses are all uncrossed, so $u$ has crossing-weighted degree at least $(d-k)+k=d$.

The matching bound now follows by applying Lemma 2 to drawing $J^{-}$and combining it with all other inequalities we derived earlier. We actually need a slightly modified version of Lemma 2 ,

Lemma $7([5])$. Let $G$ be a 1-planar graph and $A$ be a non-empty independent set in $G$ where all vertices of $A$ have degree 3 or more. Let $W_{d}$ be the vertices in $A$ that have crossingweighted degree $d$. Assume that $G$ is either simple or it has no empty lens and for any 
multiple edge at most one copy is uncrossed. Then

$$
2\left|W_{3}\right|+2\left|W_{4}\right|+\sum_{d \geq 5}(3 d-12)\left|W_{d}\right| \leq 12|V \backslash A|-24
$$

(The lemma is stated in [5] only for simple graphs, but as pointed out in a later section of [5] it holds for non-simple graphs under the above assumption.)

The proof of Lemma 4 is now finished as follows. The vertices in $F_{H} \cup T_{\sigma} \cup \bigcup_{d \geq 6} U^{d}$ are independent in $J^{-}$because all neighbours of $F_{H}$ are in $S$ and we deleted all $U T_{\sigma}$-edges during the transformations. They also have degree 3 or more by choice of $T_{\sigma}$ and Observation 6 . The remaining vertices of $J^{-}$are $S$. Using Lemma 7 we get

$$
\begin{aligned}
12|S|-24 & \geq 2\left|F_{H}\right|+2\left|T_{\sigma}\right|+\sum_{d \geq 6}(3 d-12)\left|U^{d}\right| \geq 2\left|F_{H}\right|+2\left|T_{\sigma}\right|+\sum_{d \geq 6}(2 d-10)\left|U^{d}\right| \\
& \geq 2\left|F_{H}\right|+2\left|T_{\sigma}\right|+2 \sum_{d \geq 6}\left|T^{d}\right|-10 \sum_{d \geq 6}\left|U^{d}\right|
\end{aligned}
$$

Rearranging and adding $10 \sum_{d \leq 5}\left|U^{d}\right| \geq 2 \sum_{d \leq 5}\left|T^{d}\right|$ gives

$$
12|S|+10 \sum_{d \geq 0}\left|U^{d}\right|-24 \geq 2\left|F_{H}\right|+2\left|T_{\sigma}\right|+2 \sum_{d \geq 1}\left|T^{d}\right|=2\left|F_{H}\right|+2\left|T_{H}\right|
$$

This proves Lemma 4 by $|U|=\sum_{d}\left|U^{d}\right|$.

\section{Summary and outlook}

In this paper, we considered how to find a large matching in a 1-planar graph with minimum degree 3. We argued that any matching without augmenting paths of length up to 9 has size at least $\frac{n+12}{7}$, which is the largest that one can hope for in a 1-planar graph with minimum degree 3. Such a matching can easily be found in linear time, even if no 1-planar drawings is known, by stopping the matching algorithm by Micali and Vazirani after a constant number of rounds.

It remains open how to find large matchings in 1-planar graphs with minimum degree $\delta>3$; we can argue some lower bounds on the size of matchings without 9-augmenting paths, but these are not tight. It would also be interesting to study other near-planar graph classes such as $k$-planar graphs (for $k>1$ ); here we do not even know what tight matching-bounds exist and much less how to find matchings of that size in linear time.

\section{References}

[1] Brenda S. Baker. Approximation algorithms for NP-complete problems on planar graphs. Journal of the ACM, 41(1):153-180, 1994. 
[2] Holger Bast, Kurt Mehlhorn, Guido Schäfer, and Hisao Tamaki. Matching algorithms are fast in sparse random graphs. Theory of Computing Systems, 39(1):3-14, 2006.

[3] Claude Berge. Graphs and Hypergraphs, 2nd edition. North-Holland, 1976. Translated from Graphes et Hypergraphes, Dunod, 1970.

[4] Therese Biedl, Prosenjit Bose, Erik D. Demaine, and Anna Lubiw. Efficient algorithms for Petersen's matching theorem. Journal of Algorithms, 38(1):110-134, 2001.

[5] Therese Biedl and John Wittnebel. Matchings in 1-planar graphs with large minimum degree, 2019. CoRR 1911.04603.

[6] Kellogg S. Booth and George S. Lueker. Testing for the consecutive ones property, interval graphs and graph planarity using PQ-tree algorithms. Journal of Computing and System Sciences, 13:335-379, 1976.

[7] Norishige Chiba and Takao Nishizeki. The Hamiltonian cycle problem is linear-time solvable for 4-connected planar graphs. Journal of Algorithms, 10(2):187-211, 1989.

[8] Richard Cole, Kirstin Ost, and Stefan Schirra. Edge-coloring bipartite multigraphs in $O(E \log D)$ time. Combinatorica, 21(1):5-12, 2001.

[9] Erik D. Demaine, Fedor V. Fomin, Mohammadtaghi Hajiaghayi, and Dimitrios M. Thilikos. Subexponential parameterized algorithms on bounded-genus graphs and $\mathrm{H}-$ minor-free graphs. Journal of the ACM, 52(6):866-893, 2005.

[10] Reinhard Diestel. Graph Theory, 4th Edition, volume 173 of Graduate texts in mathematics. Springer, 2012.

[11] Jack Edmonds. Maximum matchings and a polyhedron with 0,1-vertices. Journal of Research of the National Bureau of Standards, 69B:125-130, 1965.

[12] Robert Franke, Ignaz Rutter, and Dorothea Wagner. Computing large matchings in planar graphs with fixed minimum degree. Theoretical Computer Science, 412(32):40924099, 2011.

[13] Alexander Grigoriev and Hans L. Bodlaender. Algorithms for graphs embeddable with few crossings per edge. Algorithmica, 49(1):1-11, 2007.

[14] Marshall Hall, Jr. An algorithm for distinct representatives. American Mathematical Monthly, 63:716-717, 1956.

[15] John E. Hopcroft and Richard M. Karp. An $n^{5 / 2}$ algorithm for maximum matchings in bipartite graphs. SIAM Journal on Computing, 2:225-231, 1973.

[16] John E. Hopcroft and Robert E. Tarjan. Efficient planarity testing. Journal of the ACM, 21(4):549-568, 1974. 
[17] Stephen G. Kobourov, Giuseppe Liotta, and Fabrizio Montecchiani. An annotated bibliography on 1-planarity. Computer Science Review, 25:49-67, 2017.

[18] Laszlo Lovász and Michael D. Plummer. Matching theory. North-Holland Publishing Co., Amsterdam, 1986. Annals of Discrete Mathematics, 29.

[19] Silvio Micali and Vijay V. Vazirani. An $O(\sqrt{|V|}|E|)$ Algoithm for Finding Maximum Matching in General Graphs. In Proceedings of the 21st Annual Symposium on Foundations of Computer Science (FOCS'80), pages 17-27. IEEE Computer Society, 1980.

[20] Takao Nishizeki and Ilker Baybars. Lower bounds on the cardinality of the maximum matchings of planar graphs. Discrete Mathematics, 28(3):255-267, 1979.

[21] Julius Petersen. Die Theorie der regulären graphs (The theory of regular graphs). Acta Mathematica, 15:193-220, 1891.

[22] Marcus Schaefer. Crossing Numbers of Graphs. CRC Press, 2017.

[23] William T. Tutte. A theorem on planar graphs. Transactions of the American Mathematical Society, 82:99-116, 1956.

[24] Vijay V. Vazirani. A simplification of the MV matching algorithm and its proof. CoRR, abs/1210.4594, 2012. 